\title{
Fractional singular Sturm-Liouville problems on the half-line
}

\section{Pisamai Kittipoom ${ }^{*}$ (1]}

\section{"Correspondence:}

pisamai.k@psu.ac.th

Applied Analysis Research Unit, Department of Mathematics and

Statistics, Faculty of Science, Prince of Songkla University, Hat Yai,

Songkhla 90110, Thailand

\begin{abstract}
In this paper, we consider two types of singular fractional Sturm-Liouville operators. One comprises the composition of left-sided Caputo and left-sided Riemann-Liouville derivatives of order $\alpha \in(0,1)$. The other one is the composition of left-sided Riemann-Liouville and right-sided Caputo derivatives. The reality of the corresponding eigenvalues and the orthogonality of the eigenfunctions are proved. Furthermore, we formulate the fractional Laguerre Strum-Liouville problems and derive the explicit eigenfunctions as the non-polynomial functions related to Laguerre polynomials. Finally, we introduce the generalized Laguerre transform and employ it to solve the unbounded space-fractional diffusion equations.
\end{abstract}

Keywords: fractional Sturm-Liouville problem; Riemann-Liouville derivative; Caputo derivative; Weyl fractional derivative; fractional Laguerre equation; space-fractional diffusion equation

\section{Introduction}

The Sturm-Liouville problem (SLP) is a famous boundary value problem which is widely studied in pure and applied mathematics, physics, and other branches of science and engineering. However, many physical phenomena can be accurately described by differential equations with non-integer derivatives (for more details, see [1-3]). Consequently, researchers have become more interested in formulating the notion of a fractional SturmLiouville Problem (FSLP).

In [4-7], the authors defined fractional Sturm-Liouville operators (FSLOs) in different types of fractional operators. Klimek and Agrawal [4] introduced two types of FSLOs, including the composition of right-sided Caputo and left-sided Riemann-Liouville derivatives and the composition of right-sided Caputo and left-sided Riemann-Liouville derivatives. In addition, in the subsequent article [5], they presented not only eigenproperties of the FSLOs, but they also derived the explicit eigenfunctions and the corresponding eigenvalues. Concurrently, Zayernouri and Karniadakis [6] considered different FSLOs, one involving the composition of right-sided Riemann-Liouville and left-sided Caputo derivatives and the other one involving the composition of left-sided Riemann-Liouville and right-sided Caputo derivatives. In particular, they also obtained the analytical eigensolutions to the FSLPs and demonstrated the orthogonal completeness of the corresponding system of eigenfunctions. From a complementary point of view, it is known that a certain boundary value problem can be solved using a particular type of integral transform hav-

(c) The Author(s) 2017. This article is distributed under the terms of the Creative Commons Attribution 4.0 International License (http://creativecommons.org/licenses/by/4.0/), which permits unrestricted use, distribution, and reproduction in any medium, provided you give appropriate credit to the original author(s) and the source, provide a link to the Creative Commons license, and indicate if changes were made. 
ing an appropriate kernel. Recently, Ansari [8] introduced the fractional Sturm-Liouville transforms in which the kernels are the normalized eigenfunctions of the associated FSLPs on finite intervals. Bas and Metin [9] introduced the FSLO which has Coulomb potential. In [10], Bas presented the singular SLP related to the Bessel operator and investigated the properties of the eigenvalue and eigenfunctions. Also, Bas et al. [11] introduced fractional solutions of the non-homogeneous and homogeneous Bessel equation by using an $N$-fractional calculus operator. Zhang and Yi [12] presented eigensolutions to the timefractional sub-diffusion equations on fractals.

In most studies, the authors considered FSLPs on a bounded domain and the corresponding FSLOs which do not include the classical Sturm-Liouville Operators (SLOs) with integer order. Rivero et al. [7] proposed new classes of FSLOs in which the classical SLOs with integer order are included. Recently, Khosravian-Arab et al. [13] introduced FSLOs comprising infinite right-sided Riemann-Liouville and left-sided RiemannLiouville derivatives and formulated two classes of FSLPs on the half-line. Analogous to the studies of Klimek and Agrawal [4] and Zayernouri and Karniadakis [6], they derive an explicit form for the eigenfunctions as the generalized associated Laguerre functions and analyze some properties of the eigensolutions. In order to develop the spectral theory or the numerical approximation for the FSLP, a self-adjoint operator plays a crucial role. However, the FSLOs comprising infinite right-sided Riemann-Liouville and left-sided Riemann-Liouville derivatives in [13] are not self-adjoint.

In this study, we propose four types of FSLOs, which include the composition of Caputo and Riemann-Liouville derivatives and are self-adjoint. In particular, the purpose of this paper is threefold. The first purpose is to consider FSLPs on the unbounded interval $[0, \infty)$. We establish four classes of FSLPs and investigate the eigenfunctions and the eigenvalues associated to those fractional operators. Also, we show that the corresponding eigenfunctions form an orthogonal basis for the weighted Hilbert space of squareintegrable functions. The second one is to analyze the approximation properties by the generalized Laguerre function. With the objective of applying the FSLPs to fractional partial differential equations, in the third part, we introduce the generalized Laguerre transform and demonstrate its basic properties. Finally, we show that the generalized Laguerre transform can be effectively used to solve the semi-infinite space-fractional heat equation.

\section{Preliminary}

We start with some definitions of fractional integrals and derivatives and present an auxiliary lemma.

Definition 2.1 The left-sided Riemann-Liouville fractional integral with order $\alpha>0$ of the function $f$ is given by

$$
{ }_{a} I_{x}^{\alpha} f(x)=\frac{1}{\Gamma(\alpha)} \int_{a}^{x}(x-y)^{\alpha-1} f(y) d y, \quad x>a .
$$

The right-sided Riemann-Liouville fractional integral with order $\alpha>0$ of the function $f$ is given by

$$
{ }_{x} I_{b}^{\alpha} f(x)=\frac{1}{\Gamma(\alpha)} \int_{x}^{b}(y-x)^{\alpha-1} f(y) d y, \quad x<b .
$$


Definition 2.2 For a given real number $\alpha \in(n-1, n)$ with $n \in \mathbb{N}$, the left- and right-sided Riemann-Liouville fractional derivatives with order $\alpha>0$ of the function $f$ are given by

$$
\begin{aligned}
& { }_{a}^{R} D_{x}^{\alpha} f(x)=D^{n}{ }_{a} I_{x}^{n-\alpha} f(x), \quad x>a, \\
& { }_{x}^{R} D_{b}^{\alpha} f(x)=(-1)^{n} D^{n}{ }_{x} I_{b}^{n-\alpha} f(x), \quad x<b,
\end{aligned}
$$

where $D^{n}=\frac{d^{n}}{d x^{n}}$.

Analogously, by commuting the derivative and fractional integral operators in (3) and (4), we define the left- and right-sided Caputo fractional derivatives in the following.

Definition 2.3 For a given real number $\alpha \in(n-1, n)$ with $n \in \mathbb{N}$, the left- and right-sided Caputo derivatives with order $\alpha>0$ of the function $f$ are given by

$$
\begin{aligned}
& { }_{a}^{C} D_{x}^{\alpha} f(x)={ }_{a} I_{x}^{n-\alpha} D^{n} f(x), \quad x>a, \\
& { }_{x}^{C} D_{b}^{\alpha} f(x)={ }_{x} I_{b}^{n-\alpha} D^{n} f(x), \quad x<b .
\end{aligned}
$$

Definition 2.4 The infinite right-sided Riemann-Liouville fractional integral of order $\alpha>$ 0 of the function $f$ on the half-line is defined by

$$
{ }_{x} I_{\infty}^{\alpha} f(x)=\frac{1}{\Gamma(\alpha)} \int_{x}^{\infty}(y-x)^{\alpha-1} f(y) d y .
$$

Definition 2.5 Let $\alpha \in(n-1, n)$ with $n \in \mathbb{N}$. Then the infinite left-sided Riemann-Liouville and Caputo fractional derivatives of order $\alpha$ of the function $f$ on the half-line are defined by

$$
\begin{aligned}
& { }_{x}^{R} D_{\infty}^{\alpha} f(x)=(-1)^{n} D^{n}{ }_{x} I_{\infty}^{n-\alpha} f(x), \\
& { }_{x}^{C} D_{\infty}^{\alpha} f(x)={ }_{x} I_{\infty}^{n-\alpha} D^{n} f(x) .
\end{aligned}
$$

We have the following relationship between the Riemann-Liouville fractional derivative and the Caputo fractional derivative, which will be used later:

$$
{ }_{a}^{R} D_{x}^{\alpha} f(x)={ }_{a}^{C} D_{x}^{\alpha} f(x)+\sum_{k=0}^{n-1} \frac{x^{k-\alpha}}{\Gamma(k-\alpha+1)} f^{(k)}(0),
$$

for $x>0$ and $\alpha \in(n-1, n)$.

The following lemma exhibits the equations which are similar to the equations of integration by parts.

Lemma 2.6 For $\alpha \in(0,1)$, the operators ${ }_{x}^{R} D_{\infty}^{\alpha},{ }_{0}^{C} D_{x}^{\alpha},{ }_{x}^{C} D_{\infty}^{\alpha}$, and ${ }_{0}^{R} D_{x}^{\alpha}$ satisfy the following integration by parts equations:

$$
\begin{aligned}
& \int_{0}^{\infty} f(x){ }_{x}^{R} D_{\infty}^{\alpha} g(x) d x=\int_{0}^{\infty} g(x){ }_{0}^{C} D_{x}^{\alpha} f(x) d x-\left.f(x){ }_{x} I_{\infty}^{1-\alpha} g(x)\right|_{x=0} ^{\infty}, \\
& \int_{0}^{\infty} f(x){ }_{x}^{C} D_{\infty}^{\alpha} g(x) d x=\left.g(x){ }_{0} I_{x}^{1-\alpha} f(x)\right|_{x=0} ^{\infty}-\int_{0}^{\infty} g(x){ }_{0}^{R} D_{x}^{\alpha} f(x) d x .
\end{aligned}
$$


Proof By definition,

$$
\int_{0}^{\infty} f(x){ }_{x}^{R} D_{\infty}^{\alpha} g(x) d x=-\int_{0}^{\infty} f(x) \frac{d}{d x}\left({ }_{x} I_{\infty}^{1-\alpha} g(x)\right) d x .
$$

Using the integration by parts equation gives

$$
\begin{aligned}
\int_{0}^{\infty} f(x){ }_{x}^{R} D_{\infty}^{\alpha} g(x) d x & =-\left.f(x)_{x} I_{\infty}^{1-\alpha} g(x)\right|_{x=0} ^{\infty}+\int_{0}^{\infty} f^{\prime}(x)_{x} I_{\infty}^{1-\alpha} g(x) d x \\
& =-\left.f(x)_{x} I_{\infty}^{1-\alpha} g(x)\right|_{x=0} ^{\infty}+\frac{1}{\Gamma(1-\alpha)} \int_{0}^{\infty} f^{\prime}(x) \int_{x}^{\infty}(y-x)^{-\alpha} g(y) d y d x .
\end{aligned}
$$

By reversing the order of integration, we get

$$
\begin{aligned}
\int_{0}^{\infty} f(x){ }_{x}^{R} D_{\infty}^{\alpha} g(x) d x & =-\left.f(x)_{x} I_{\infty}^{1-\alpha} g(x)\right|_{x=0} ^{\infty}+\frac{1}{\Gamma(1-\alpha)} \int_{0}^{\infty} g(y) \int_{0}^{y} f^{\prime}(x)(y-x)^{-\alpha} d x d y \\
& =-\left.f(x)_{x} I_{\infty}^{1-\alpha} g(x)\right|_{x=0} ^{\infty}+\frac{1}{\Gamma(1-\alpha)} \int_{0}^{\infty} g(y)_{0} I_{y}^{1-\alpha} f^{\prime}(y) d y \\
& =-\left.f(x)_{x} I_{\infty}^{1-\alpha} g(x)\right|_{x=0} ^{\infty}+\int_{0}^{\infty} g(y){ }_{0}^{C} D_{y}^{\alpha} f(y) d y,
\end{aligned}
$$

which proves (11).

Equality (12) can be proved similarly. Again, by definition,

$$
\int_{0}^{\infty} g(x){ }_{0}^{R} D_{x}^{\alpha} f(x) d x=\int_{0}^{x} g(x) \frac{d}{d x}\left({ }_{0} I_{x}^{1-\alpha} f(x)\right) d x .
$$

Applying the integration by parts equation and reversing the order of integration yields

$$
\begin{aligned}
\int_{0}^{\infty} g(x){ }_{0}^{R} D_{x}^{\alpha} f(x) d x & =\left.g(x)_{0} I_{x}^{1-\alpha} f(x)\right|_{0} ^{x}-\int_{0}^{\infty} g^{\prime}(x)_{0} I_{x}^{1-\alpha} f(x) d x \\
& =\left.g(x)_{0} I_{x}^{1-\alpha} g(x)\right|_{0} ^{x}-\frac{1}{\Gamma(1-\alpha)} \int_{0}^{x} g^{\prime}(x) \int_{0}^{x}(x-y)^{-\alpha} f(y) d y d x \\
& =\left.g(x)_{0} I_{x}^{1-\alpha} g(x)\right|_{0} ^{x}-\frac{1}{\Gamma(1-\alpha)} \int_{0}^{\infty} f(y) \int_{x}^{\infty} g^{\prime}(x)(x-y)^{-\alpha} d x d y \\
& =\left.g(x)_{0} I_{x}^{1-\alpha} g(x)\right|_{0} ^{x}-\int_{0}^{x} f(y)_{x}^{C} D_{\infty}^{\alpha} g(y) d y .
\end{aligned}
$$

\section{Singular fractional Sturm-Liouville problems}

In this section, we introduce four types of non-local differential operators comprising the $\mu$ th-order Caputo fractional derivative and the $\mu$ th-order Riemann-Liouville fractional derivatives for $\mu \in(0,1) . \mathbf{L}_{i}^{\mu}: \mathcal{D}_{i} \rightarrow \mathcal{D}_{i}$ is defined by

$$
\begin{aligned}
& \mathbf{L}_{1}^{\mu}={ }_{x} D_{\infty}^{\mu}\left[p_{1}(x){ }_{0}^{R} D_{x}^{\mu}(\cdot)\right]+\lambda \omega_{\mu}^{(1)}(x) f(x), \quad 0 \leq x<\infty, \\
& \mathbf{L}_{2}^{\mu}={ }_{0}^{C} D_{x}^{\mu}\left[p_{2}(x){ }_{x}^{R} D_{\infty}^{\mu}(\cdot)\right]+\lambda \omega_{\mu}^{(2)}(x) f(x), \quad 0 \leq x<\infty, \\
& \mathbf{L}_{3}^{\mu}={ }_{x}^{R} D_{\infty}^{\mu}\left[p_{3}(x){ }_{0}^{C} D_{x}^{\mu}(\cdot)\right]+\lambda \omega_{\mu}^{(3)}(x) f(x), \quad 0 \leq x<\infty, \\
& \mathbf{L}_{4}^{\mu}={ }_{0}^{R} D_{x}^{\mu}\left[p_{4}(x){ }_{x}^{C} D_{\infty}^{\mu}(\cdot)\right]+\lambda \omega_{\mu}^{(4)}(x) f(x), \quad 0 \leq x<\infty,
\end{aligned}
$$


where $p_{i}, \omega_{i}^{(\mu)}, i=1, \ldots, 4$ are real valued continuous functions on $[0, \infty)$, and

$$
\begin{aligned}
& \mathcal{D}_{1}=\left\{u: u(x)=x^{\alpha+\mu-1} v(x), v \in L_{\omega_{\mu}^{(1)}}^{2}(0, \infty), u \text { satisfies the below condition (18) }\right\}, \\
& \mathcal{D}_{2}=\left\{u: u(x)=e^{-x} v(x), v \in L_{\omega_{\mu}^{(2)}}^{2}(0, \infty), u \text { satisfies the below condition }(20)\right\}, \\
& \mathcal{D}_{3}=\left\{u: u(x)=x^{\alpha+\mu-1} v(x), v \in L_{\omega_{\mu}^{(3)}}^{2}(0, \infty), u \text { satisfies the below condition (22) },\right. \\
& \mathcal{D}_{4}=\left\{u: u(x)=e^{-x} v(x), v \in L_{\omega_{\mu}^{(4)}}^{2}(0, \infty), u \text { satisfies the below condition }(24)\right\} .
\end{aligned}
$$

In this study, we consider four classes of singular fractional SLPs (SFSLPs):

SFSLP1: $\quad{ }_{x}^{C} D_{\infty}^{\mu}\left[p_{1}(x){ }_{0}^{R} D_{x}^{\mu} f(x)\right]+\lambda \omega_{\mu}^{(1)}(x) f(x)=0, \quad 0 \leq x<\infty$,

subject to

$$
\begin{aligned}
& \left.p_{1}(x)_{0} I_{x}^{1-\mu} f(x)\right|_{x=0}=0, \\
& \lim _{x \rightarrow \infty} p_{1}(x)_{0} I_{x}^{1-\mu} f(x)=0,
\end{aligned}
$$

SFSLP2: $\quad{ }_{0}^{C} D_{x}^{\mu}\left[p_{2}(x){ }_{x}^{R} D_{\infty}^{\mu} f(x)\right]+\lambda \omega_{\mu}^{(2)}(x) f(x)=0, \quad 0 \leq x<\infty$,

subject to

$$
\begin{aligned}
& \left.p_{2}(x)_{x} I_{\infty}^{1-\mu} f(x)\right|_{x=0}=0, \\
& \lim _{x \rightarrow \infty}{ }_{x} D_{\infty}^{\mu} f(x)=0,
\end{aligned}
$$

SFSLP3: $\quad{ }_{x}^{R} D_{\infty}^{\mu}\left[p_{3}(x){ }_{0}^{C} D_{x}^{\mu} f(x)\right]+\lambda \omega_{\mu}^{(3)}(x) f(x)=0, \quad 0 \leq x<\infty$,

subject to

$$
\begin{aligned}
& f(0)=0, \\
& \lim _{x \rightarrow \infty}{ }_{x} I_{\infty}^{1-\mu}\left[p_{3}(x){ }_{0}^{C} D_{x}^{\mu} f(x)\right]=0,
\end{aligned}
$$

SFSLP4: $\quad{ }_{0}^{R} D_{x}^{\mu}\left[p_{4}(x){ }_{x}^{C} D_{\infty}^{\mu} f(x)\right]+\lambda \omega_{\mu}^{(4)}(x) f(x)=0, \quad 0 \leq x<\infty$,

subject to

$$
\begin{aligned}
& \left.{ }_{0} I_{x}^{1-\mu}\left[p_{4}(x){ }_{x}^{C} D_{\infty}^{\mu} f(x)\right]\right|_{x=0}=0, \\
& \lim _{x \rightarrow \infty} f(x)=0 .
\end{aligned}
$$

In the following theorem, we present the self-adjointness of FSLOs defined in (13), (14), (15), and (16).

Theorem 3.1 The FSLOs $\mathbf{L}_{i}^{\mu}$ defined in (13), (14), (15), and (16) are self-adjoint on $\mathcal{D}_{i}$, $i=1, \ldots, 4$.

Proof First, let $f$ and $g$ be arbitrary real valued functions satisfying boundary conditions (18). Then we have

$$
\left\langle\mathbf{L}_{1}^{\mu} f, g\right\rangle-\left\langle f, \mathbf{L}_{1}^{\mu} g\right\rangle=\int_{0}^{\infty}{ }_{x}^{C} D_{\infty}^{\mu}\left[p_{1}(x){ }_{0}^{R} D_{x}^{\mu} f(x)\right] g(x) d x-\int_{0}^{\infty} f(x){ }_{x}^{C} D_{\infty}^{\mu}\left[p_{1}(x){ }_{0}^{R} D_{x}^{\mu} g(x)\right] d x .
$$


Using the integration by parts (12) and applying the boundary conditions (18), we get

$$
\begin{aligned}
\left\langle\mathbf{L}_{1}^{\mu} f, g\right\rangle-\left\langle f, \mathbf{L}_{1}^{\mu} g\right\rangle= & \left.p_{1}(x)_{0}^{R} D_{x}^{\mu} f(x)_{0} I_{x}^{1-\mu} g(x)\right|_{0} ^{\infty}-\int_{0}^{\infty} p_{1}(x)_{0}^{R} D_{x}^{\mu} f(x){ }_{0}^{R} D_{x}^{\mu} g(x) d x \\
& -\left[\left.p_{1}(x)_{0}^{R} D_{x}^{\mu} g(x)_{0} I_{x}^{1-\mu} f(x)\right|_{0} ^{\infty}-\int_{0}^{\infty} p_{1}(x)_{0}^{R} D_{x}^{\mu} g(x)_{0}^{R} D_{x}^{\mu} f(x) d x\right] \\
= & 0 .
\end{aligned}
$$

Second, let $f$ and $g$ be arbitrary real valued functions satisfying boundary conditions (20). Then we have

$$
\left\langle\mathbf{L}_{2}^{\mu} f, g\right\rangle-\left\langle f, \mathbf{L}_{2}^{\mu} g\right\rangle=\int_{0}^{\infty}{ }_{0}^{C} D_{x}^{\mu}\left[p_{2}(x){ }_{x}^{R} D_{\infty}^{\mu} f(x)\right] g(x) d x-\int_{0}^{\infty} f(x){ }_{0}^{C} D_{x}^{\mu}\left[p_{2}(x){ }_{x}^{R} D_{\infty}^{\mu} g(x)\right] d x .
$$

Using the integration by parts (11) and applying the boundary conditions (20), we get

$$
\begin{aligned}
\left\langle\mathbf{L}_{2}^{\mu} f, g\right\rangle-\left\langle f, \mathbf{L}_{2}^{\mu} g\right\rangle= & \int_{0}^{\infty} p_{2}(x)_{x}^{R} D_{\infty}^{\mu} f(x){ }_{x}^{R} D_{\infty}^{\mu} g(x) d x+\left.p_{2}(x){ }_{x}^{R} D_{\infty}^{\mu} f(x) I_{x}^{1-\mu} g(x)\right|_{0} ^{\infty} \\
& -\left[\int_{0}^{\infty} p_{2}(x)_{x}^{R} D_{\infty}^{\mu} g(x){ }_{x}^{R} D_{\infty}^{\mu} f(x) d x+\left.p_{2}(x)_{x}^{R} D_{\infty}^{\mu} g(x)_{x} I_{\infty}^{1-\mu} f(x)\right|_{0} ^{\infty}\right]
\end{aligned}
$$

$$
=0 \text {. }
$$

Third, let $f$ and $g$ be arbitrary real valued functions satisfying boundary conditions (22). Then we have

$$
\left\langle\mathbf{L}_{3}^{\mu} f, g\right\rangle-\left\langle f, \mathbf{L}_{3}^{\mu} g\right\rangle=\int_{0}^{\infty}{ }_{x}^{R} D_{\infty}^{\mu}\left[p_{3}(x){ }_{0}^{C} D_{x}^{\mu} f(x)\right] g(x) d x-\int_{0}^{\infty} f(x){ }_{x}^{R} D_{\infty}^{\mu}\left[p_{3}(x){ }_{0}^{C} D_{x}^{\mu} g(x)\right] d x .
$$

Using the integration by parts (11) and applying the boundary conditions (22), we get

$$
\begin{aligned}
\left\langle\mathbf{L}_{3}^{\mu} f, g\right\rangle-\left\langle f, \mathbf{L}_{3}^{\mu} g\right\rangle= & \left.\int_{0}^{\infty} p_{3}(x)_{0}^{C} D_{x}^{\mu} f(x){ }_{0}^{C} D_{x}^{\mu} g(x) d x-g(x)_{x} I_{\infty}^{1-\mu}\left[p_{3}(x)_{0}^{C} D_{x}^{\mu} f(x)\right)\right]\left.\right|_{0} ^{\infty} \\
& -\left[\int_{0}^{\infty} p_{3}(x)_{0}^{C} D_{x}^{\mu} g(x){ }_{0}^{C} D_{x}^{\mu} f(x) d x-\left.f(x)_{x} I_{\infty}^{1-\mu}\left[p_{3}(x)_{0}^{C} D_{x}^{\mu} g(x)\right]\right|_{0} ^{\infty}\right] \\
= & 0 .
\end{aligned}
$$

Finally, let $f$ and $g$ be arbitrary real valued functions satisfying boundary conditions (24). Then we have

$$
\left\langle\mathbf{L}_{4}^{\mu} f, g\right\rangle-\left\langle f, \mathbf{L}_{4}^{\mu} g\right\rangle=\int_{0}^{\infty}{ }_{0}^{R} D_{x}^{\mu}\left[p_{4}(x){ }_{x} D_{\infty}^{\mu} f(x)\right] g(x) d x-\int_{0}^{\infty} f(x){ }_{0}^{R} D_{x}^{\mu}\left[p_{4}(x){ }_{x} D_{\infty}^{\mu} g(x)\right] d x .
$$

Using the integration by parts (12) and applying the boundary conditions (24), we get

$$
\begin{aligned}
\left\langle\mathbf{L}_{4}^{\mu} f, g\right\rangle-\left\langle f, \mathbf{L}_{4}^{\mu} g\right\rangle= & \left.g(x)_{0} I_{x}^{1-\mu}\left[p_{4}(x)_{x}^{C} D_{\infty}^{\mu} f(x)\right)\right]\left.\right|_{0} ^{\infty}-\int_{0}^{\infty} p_{4}(x)_{x}^{C} D_{\infty}^{\mu} f(x)_{x}^{C} D_{\infty}^{\mu} g(x) d x \\
& \left.-\left.\left[f(x)_{0} I_{x}^{1-\mu}\left[p_{4}(x)_{x}^{C} D_{\infty}^{\mu} g(x)\right)\right]\right|_{0} ^{\infty}-\int_{0}^{\infty} p_{4}(x)_{x}^{C} D_{\infty}^{\mu} g(x)_{x}^{C} D_{\infty}^{\mu} f(x) d x\right]
\end{aligned}
$$$$
=0 \text {. }
$$ 
Theorem 3.2 The eigenvalues of SFSLP1 (17)-(18) are real and the corresponding eigenfunctions of the distinct eigenvalues of SFSLP1 (17)-(18) are orthogonal with respect to the weight function $\omega_{\mu}^{(1)}$.

Proof In the first part, we prove that the eigenvalues of SFSLP1 (17)-(18) are real-valued. Assume that an eigenfunction $f(x)$ and its complex conjugate $\bar{f}(x)$ correspond to eigenvalue $\lambda$ and its complex conjugate eigenvalue $\bar{\lambda}$, respectively. Then

$$
\mathbf{L}_{1}^{\mu} f(x)+\lambda \omega_{\mu}^{(1)}(x) f(x)=0,
$$

subject to the boundary conditions

$$
\begin{aligned}
& \left.p_{1}(x)_{0} I_{x}^{1-\mu} f(x)\right|_{x=0}=0, \\
& \lim _{x \rightarrow \infty} p_{1}(x)_{0} I_{x}^{1-\mu} f(x)=0,
\end{aligned}
$$

and

$$
\mathbf{L}_{1}^{\mu} \bar{f}(x)+\bar{\lambda} \omega_{\mu}^{(1)}(x) \bar{f}(x)=0,
$$

subject to the boundary conditions

$$
\begin{aligned}
& \left.p_{1}(x)_{0} I_{x}^{1-\mu} \bar{f}(x)\right|_{x=0}=0, \\
& \lim _{x \rightarrow \infty} p_{1}(x)_{0} I_{x}^{1-\mu} \bar{f}(x)=0 .
\end{aligned}
$$

By multiplying (25) by $\bar{f}$ and (27) by $f$ and then subtracting both equations, we obtain

$$
(\lambda-\bar{\lambda}) \omega_{\mu}^{(1)}(x) f(x) \bar{f}(x)=f(x) L_{1}^{\mu} \bar{f}(x)-\bar{f}(x) L_{1}^{\mu} f(x)
$$

Integrating over $[0, \infty)$ and using the integration by parts equation (12), we get

$$
\begin{aligned}
(\lambda- & \bar{\lambda}) \int_{0}^{\infty} \omega_{\mu}^{(1)}(x)|f(x)|^{2} d x \\
= & \int_{0}^{\infty} f(x) \mathbf{L}_{1}^{\mu} \bar{f}(x) d x-\int_{0}^{\infty} \bar{f}(x) \mathbf{L}_{1}^{\mu} f(x) d x \\
= & \int_{0}^{\infty} p_{1}(x){ }_{0}^{R} D_{x}^{\mu} \bar{f}(x){ }_{0}^{R} D_{x}^{\mu} f(x) d x+\left.p_{1}(x)_{0}^{R} D_{x}^{\mu} \bar{f}(x)_{0} I_{x}^{1-\mu} f(x)\right|_{x=0} ^{\infty} \\
& \quad-\int_{0}^{\infty} p_{1}(x){ }_{0}^{R} D_{x}^{\mu} f(x)_{0}^{R} D_{x}^{\mu} \bar{f}(x) d x-\left.p_{1}(x)_{0}^{R} D_{x}^{\mu} f(x)_{0} I_{x}^{1-\mu} \bar{f}(x)\right|_{x=0} ^{\infty} .
\end{aligned}
$$

Using the boundary conditions (26) and (28), we have

$$
(\lambda-\bar{\lambda}) \int_{0}^{\infty} \omega_{\mu}^{(1)}(x)|f(x)|^{2} d x=0
$$

Since $\omega_{\mu}^{(1)}$ and the eigenfunction $f$ are non-zero, we conclude that $\lambda=\bar{\lambda}$. 
Next, we prove the orthogonality of two eigenfunctions corresponding to distinct eigenvalues. Suppose $\lambda_{1}$ and $\lambda_{2}$ are distinct eigenvalues of SFSLP1 (17)-(18) and the corresponding eigenfunctions are $f_{1}$ and $f_{2}$, respectively. Similar to the first part, we write equation (17) for functions $f_{1}$ and $f_{2}$, multiply the equation for $f_{1}$ by $f_{2}$ and vice versa, and subtract one equation from another to obtain

$$
f_{1}(x) \mathbf{L}_{1}^{\mu} f_{2}(x)-f_{2}(x) \mathbf{L}_{1}^{\mu} f_{1}(x)=\left(\lambda_{1}-\lambda_{2}\right) \omega_{\mu}^{(1)}(x) f_{1}(x) f_{2}(x) .
$$

Integrating the above equation in $[0, \infty)$ and applying the boundary conditions (18) yields

$$
\left(\lambda_{1}-\lambda_{2}\right) \int_{0}^{\infty} \omega_{\mu}^{(1)}(x) f_{1}(x) f_{2}(x) d x=0 .
$$

Since $\lambda_{1} \neq \lambda_{2}$, the orthogonality is proved.

We further study the eigenproperties of SFSLP2-SFSLP4 in the following theorem. The proof is straightforward and similar to Theorem 3.2 and thus omitted.

Theorem 3.3 The eigenvalues of SFSLP2-SFSLP4 are real and the corresponding eigenfunctions of the distinct eigenvalues of SFSLP2-SFSLP4 are orthogonal with respect to the weight function $\omega_{\mu}^{(i)}, i=2,3,4$.

\section{The fractional Laguerre equation}

In this section, we consider the special singular fractional Sturm-Liouville equation, show the discreteness of the corresponding eigenvalues, and we find the explicit eigenfunctions. Before that, we recall the Laguerre equation, Laguerre polynomials, and some useful properties.

For $\alpha<1$, the equation

$$
x y^{\prime \prime}+(1-\alpha-x) y^{\prime}+\lambda y=0, \quad 0 \leq x<\infty
$$

is known as the Laguerre equation, which can be converted to a singular Sturm-Liouville form as follows:

$$
D\left(x^{1-\alpha} e^{-x} D(y(x))\right)+\lambda x^{-\alpha} e^{-x} y(x)=0 .
$$

In particular, the Laguerre polynomials, $\mathcal{L}_{n}^{(\alpha)}(x)$, defined by

$$
\mathcal{L}_{n}^{(\alpha)}(x)=\sum_{k=0}^{n}(-1)^{k}\left(\begin{array}{l}
n+\alpha \\
n-k
\end{array}\right) \frac{x^{k}}{k !}, \quad n=0,1,2, \ldots
$$

are eigenfunctions of the Laguerre equation (29) and the corresponding eigenvalues are $\lambda_{n}=-\lambda=-n$.

A useful representation of the Laguerre polynomials is the following:

$$
\mathcal{L}_{n}^{(\alpha)}(x)=\left(\begin{array}{c}
n+\alpha \\
n
\end{array}\right){ }_{1} F_{1}(-n, \alpha+1 ; x)
$$


where ${ }_{1} F_{1}(-n, \alpha+1 ; x)=\sum_{k=0}^{n}(-1)^{k} \frac{n(n+1) \cdots(n+k-1)}{(\alpha+1)(\alpha+2) \cdots(\alpha+k+1)} \frac{x^{k}}{k !}$ is the confluent hypergeometric function. The Laguerre polynomials $\mathcal{L}_{n}^{(\alpha)}(x)$ are orthogonal with respect to the weight function $\omega_{\alpha}=x^{\alpha} e^{-x}$. We have

$$
\int_{0}^{\infty} \mathcal{L}_{n}^{(\alpha)}(x) \mathcal{L}_{m}^{(\alpha)}(x) x^{\alpha} e^{-x} d x=\frac{\Gamma(n+\alpha+1)}{\Gamma(n+1)} \delta_{m n}
$$

In addition, the generating function for the Laguerre polynomials is

$$
\frac{e^{-\frac{t}{1-t} x}}{(1-t)^{\alpha+1}}=\sum_{n=0}^{\infty} t^{n} \mathcal{L}_{n}^{(\alpha)}(x), \quad|t|<1
$$

In the following, we prove several equations of fractional integrals and derivatives of the Laguerre polynomial which will be used later.

Lemma 4.1 For $n-1<\mu<n$ and for all $x \in[0, \infty)$, we have

$$
\begin{aligned}
& { }_{0} I_{x}^{\mu}\left(x^{\alpha} \mathcal{L}_{n}^{(\alpha)}(x)\right)=\frac{\Gamma(n+\alpha+1)}{\Gamma(n+\alpha+\mu+1)} x^{\alpha+\mu} \mathcal{L}_{n}^{(\alpha+\mu)}(x), \quad \alpha>-1, \\
& { }_{0}^{R} D_{x}^{\mu}\left(x^{\alpha} \mathcal{L}_{n}^{(\alpha)}(x)\right)=\frac{\Gamma(n+\alpha+1)}{\Gamma(n+\alpha-\mu+1)} x^{\alpha-\mu} \mathcal{L}_{n}^{(\alpha-\mu)}(x), \quad \alpha>\mu-1, \\
& { }_{0}^{C} D_{x}^{\mu}\left(x^{\alpha} \mathcal{L}_{n}^{(\alpha)}(x)\right)=\frac{\Gamma(n+\alpha+1)}{\Gamma(n+\alpha-\mu+1)} x^{\alpha-\mu} \mathcal{L}_{n}^{(\alpha-\mu)}(x), \quad \alpha>\mu-1 .
\end{aligned}
$$

Proof We use the identity of the fractional integral of the Laguerre polynomials exhibited in [14], p.287. For real numbers $a, b$, and $\mu$ with $b, \mu \geq 0$,

$$
x^{b+\mu-1}{ }_{1} F_{1}(a, b+\mu ; x)=\frac{\Gamma(b+\mu)}{\Gamma(b) \Gamma(\mu)} \int_{0}^{x}(x-t)^{\mu-1} t^{b-1}{ }_{1} F_{1}(a, b ; t) d t .
$$

Taking $a=-n, b=\alpha+1$, (30) implies that

$$
\begin{aligned}
& x^{\alpha+\mu}\left(\begin{array}{c}
n+\alpha \\
n
\end{array}\right) \mathcal{L}_{n}^{(\alpha+\mu)}(x)=\frac{\Gamma(\alpha+1+\mu)}{\Gamma(\alpha+1) \Gamma(\mu)} \int_{0}^{x}(x-t)^{\mu-1} t^{\alpha}\left(\begin{array}{c}
n+\alpha+\mu \\
n
\end{array}\right) \mathcal{L}_{n}^{(\alpha)}(x) d t \\
& x^{\alpha+\mu} \mathcal{L}_{n}^{(\alpha+\mu)}(x)=\frac{\Gamma(n+\alpha+1+\mu)}{\Gamma(n+\alpha+1) \Gamma(\mu)} \int_{0}^{x}(x-t)^{\mu-1} t^{\alpha} \mathcal{L}_{n}^{(\alpha)}(x) d t .
\end{aligned}
$$

The following equation holds:

$$
{ }_{0} I_{x}^{\mu}\left[x^{\alpha} \mathcal{L}_{n}^{(\alpha)}(x)\right]=\frac{\Gamma(n+\alpha+1)}{\Gamma(n+\alpha+\mu+1)} x^{\alpha+\mu} \mathcal{L}_{n}^{(\alpha+\mu)}(x)
$$

Since ${ }_{0}^{R} D_{x 0}^{\mu} I_{x}^{\mu} f(x)=f(x)$, we derive (34) by performing ${ }_{0}^{R} D_{x}^{\mu}$ on both sides of the last equation and taking $\alpha+\mu$ by $\alpha$. We have, for $\alpha>\mu-1$,

$$
{ }_{0}^{R} D_{x}^{\mu}\left(x^{\alpha} \mathcal{L}_{n}^{(\alpha)}(x)\right)=\frac{\Gamma(n+\alpha+1)}{\Gamma(n+\alpha-\mu+1)} x^{\alpha-\mu} \mathcal{L}_{n}^{(\alpha-\mu)}(x), \quad \alpha>\mu-1 .
$$

Since $\left.x^{\alpha} \mathcal{L}_{n}^{(\alpha)}(x)\right|_{x=0}=0$, by (10) we replace ${ }_{0}^{C} D_{x}^{\mu}$ by ${ }_{0}^{R} D_{x}^{\mu}$ and complete the proof of (35). 
Lemma 4.2 For $n-1<\mu<n$ and for all $x \in[0, \infty)$, we have

$$
\begin{aligned}
& { }_{x} I_{\infty}^{\mu}\left(e^{-x} \mathcal{L}_{n}^{(\alpha)}(x)\right)=e^{-x} \mathcal{L}_{n}^{(\alpha-\mu)}(x), \quad \alpha>\mu-1, \\
& { }_{x}^{R} D_{\infty}^{\mu}\left(e^{-x} \mathcal{L}_{n}^{(\alpha)}(x)\right)=(-1)^{n} e^{-x} \mathcal{L}_{n}^{(\alpha+\mu)}(x), \quad \alpha>-1, \\
& { }_{x} D_{\infty}^{\mu}\left(e^{-x} \mathcal{L}_{n}^{(\alpha)}(x)\right)=(-1)^{n} e^{-x} \mathcal{L}_{n}^{(\alpha+\mu)}(x), \quad \alpha>-1 .
\end{aligned}
$$

Proof Once again, we use the equation in [14], p.287:

$$
e^{-x} \mathcal{L}_{n}^{(\alpha)}(x)=\frac{1}{\Gamma(\beta-\alpha)} \int_{x}^{\infty}(t-x)^{\beta-\alpha-1} e^{-t} \mathcal{L}_{n}^{(\beta)}(t) d t, \quad \beta>\alpha>-1 .
$$

Taking $\beta=\alpha+\mu$, we get

$$
e^{-x} \mathcal{L}_{n}^{(\alpha)}(x)={ }_{x} I_{\infty}^{\mu}\left(e^{-x} \mathcal{L}_{n}^{(\alpha+\mu)}(x)\right), \quad \alpha+\mu>\mu-1 .
$$

By choosing $\alpha+\mu$ by $\alpha$, we prove equation (36) when $\alpha>\mu-1$.

Using the fact that ${ }_{x}^{R} D_{\infty}^{\mu} I_{\infty}^{\mu} f(x)=(-1)^{n} f(x)$, we derive equation (37).

By definition, using (37) and (36), we have

$$
\begin{aligned}
{ }_{x} D_{\infty}^{\mu}\left(e^{-x} \mathcal{L}_{n}^{(\alpha)}(x)\right) & ={ }_{x} I_{\infty}^{1-\mu}\left[\frac{d}{d x}\left(e^{-x} \mathcal{L}_{n}^{(\alpha)}(x)\right)\right] \\
& =(-1)^{n}{ }_{x} I_{\infty}^{1-\mu}\left[e^{-x} \mathcal{L}_{n}^{(\alpha)}(x)\right] \\
& =(-1)^{n} e^{-x} \mathcal{L}_{n}^{(\alpha)}(x),
\end{aligned}
$$

which proves (38).

Now, we consider SFSLP1 (17)-(18) with $p_{1}(x)=x^{1-\alpha} e^{-x}$ and the weight function $\omega_{1}^{(\mu)}(x)=$ $x^{1-\alpha-\mu} e^{-x}$. For the parameters $\alpha, \mu \in(0,1)$, we obtain

$$
{ }_{x}^{C} D_{\infty}^{\mu}\left(x^{1-\alpha} e^{-x}{ }_{0}^{R} D_{x}^{\mu} \Phi(x)\right)+\lambda x^{1-\alpha-\mu} e^{-x} \Phi(x)=0, \quad 0 \leq x<\infty,
$$

subject to

$$
\begin{aligned}
& \left.x^{1-\alpha} e^{-x}{ }_{0}^{1-\mu} \Phi(x)\right|_{x=0}=0, \\
& \lim _{x \rightarrow \infty} x^{1-\alpha} e^{-x} I_{x}^{1-\mu} \Phi(x)=0 .
\end{aligned}
$$

We derive the eigenfunctions of SFSLP1 (39)-(40) and the corresponding eigenvalues, which agree well with Theorem 3.2.

Theorem 4.3 The eigenvalues of SFSLP1 (39)-(40) are given by

$$
\lambda_{n}^{(\alpha, \mu)}=-\frac{\Gamma(n+\alpha+\mu)}{\Gamma(n+\alpha)}, \quad n=0,1,2, \ldots
$$

The corresponding eigenfunctions to different eigenvalues are orthogonal with respect to the weighted functions, $\omega_{1}^{(\alpha, \mu)}(x)=x^{1-\alpha-\mu} e^{-x}$, and are given by

$$
\Phi_{n}^{(\alpha, \mu)}(x)=x^{\alpha+\mu-1} \mathcal{L}_{n}^{(\alpha+\mu-1)}(x), \quad n=0,1,2, \ldots
$$


Proof First we prove that the generalized Laguerre polynomials (41) obey the boundary conditions (40). Using (33), we have

$$
\begin{aligned}
x^{1-\alpha} e^{-x} I_{x}^{1-\mu}\left[x^{\alpha+\mu-1} \mathcal{L}_{n}^{(\alpha+\mu-1)}(x)\right] & =x^{1-\alpha} e^{-x} x^{\alpha+\mu-1+(1-\mu)} \mathcal{L}_{n}^{(\alpha+\mu-1+(1-\mu))}(x) \\
& =\frac{\Gamma(n+\alpha+\mu)}{\Gamma(n+\alpha+1)} x e^{-x} \mathcal{L}_{n}^{(\alpha)}(x) .
\end{aligned}
$$

Hence,

$$
\left.x^{1-\alpha} e^{-x} I_{x}^{1-\mu}\left[x^{\alpha+\mu-1} \mathcal{L}_{n}^{(\alpha+\mu-1)}(x)\right]\right|_{x=0}=0
$$

and

$$
\lim _{x \rightarrow \infty} x^{1-\alpha} e^{-x} I_{x}^{1-\mu}\left[x^{\alpha+\mu-1} \mathcal{L}_{n}^{(\alpha+\mu-1)}(x)\right]=0 .
$$

Next, substituting the function $\Phi_{n}^{(\alpha, \mu)}$ in (41) in equation (39) yields

$$
{ }_{x}^{C} D_{\infty}^{\mu}\left[x^{1-\alpha} e^{-x}{ }_{0}^{R} D_{x}^{\mu}\left(x^{\alpha+\mu-1} \mathcal{L}_{n}^{(\alpha+\mu-1)}(x)\right)\right]=-\lambda e^{-x} \mathcal{L}_{n}^{(\alpha+\mu-1)}(x) .
$$

Thanks to (34), (37), and (36), we have

$$
\begin{aligned}
{ }_{x}^{C} D_{\infty}^{\mu}\left[x^{1-\alpha} e^{-x}{ }_{0}^{R} D_{x}^{\mu}\left(x^{\alpha+\mu-1} \mathcal{L}_{n}^{(\alpha+\mu-1)}(x)\right)\right] & =\frac{\Gamma(n+\alpha+\mu)}{\Gamma(n+\alpha)}{ }_{x} D_{\infty}^{\mu}\left[e^{-x} \mathcal{L}_{n}^{(\alpha-1)}(x)\right] \quad \text { (by (34)) } \\
& =-\frac{\Gamma(n+\alpha+\mu)}{\Gamma(n+\alpha)} e^{-x} \mathcal{L}_{n}^{(\alpha+\mu-1)}(x) \quad(\text { by }(38)) \\
& =-\lambda_{n}^{(\alpha, \mu)} \omega_{1}^{(\alpha, \mu)}(x) \Phi_{n}^{(\alpha, \mu)}(x),
\end{aligned}
$$

where the corresponding eigenvalues are given by

$$
\lambda_{n}^{(\alpha, \mu)}=\frac{\Gamma(n+\alpha+\mu)}{\Gamma(n+\alpha)} .
$$

Finally, we show that the eigenfunctions (41) are orthogonal with respect to $\omega_{1}^{(\alpha, \mu)}(x)=$ $x^{1-\alpha-\mu} e^{-x}$ as follows:

$$
\begin{aligned}
\int_{0}^{\infty} \Phi_{m}^{(\alpha, \mu)}(x) \Phi_{n}^{(\alpha, \mu)}(x) \omega^{\alpha, \mu}(x) d x & =\int_{0}^{\infty} x^{2(\alpha+\mu-1)} \mathcal{L}_{n}^{(\alpha+\mu-1)}(x) \mathcal{L}_{m}^{(\alpha+\mu-1)}(x) x^{1-\alpha-\mu} e^{-x} d x \\
& =\int_{0}^{\infty} \mathcal{L}_{n}^{(\alpha+\mu-1)}(x) \mathcal{L}_{m}^{(\alpha+\mu-1)}(x) x^{\alpha+\mu-1} e^{-x} d x \\
& =\frac{\Gamma(n+\alpha+\mu)}{\Gamma(n+1)} \delta_{m n} .
\end{aligned}
$$

We now turn to SFSLP2 (19)-(20) with $p_{2}(x)=x^{\alpha+\mu-1} e^{x}$ and the weight function $\omega_{2}^{(\alpha)}(x)=$ $x^{\alpha-1} e^{x}$. For the parameter $\alpha, \mu \in(0,1)$, we obtain

$$
{ }_{0}^{C} D_{x}^{\mu}\left(x^{\alpha+\mu-1} e^{x}{ }_{x}^{R} D_{\infty}^{\mu} \Psi(x)\right)+\lambda x^{\alpha-1} e^{x} \Psi(x)=0, \quad 0 \leq x<\infty,
$$


subject to

$$
\begin{aligned}
& \left.x^{\alpha+\mu-1} e^{x}{ }_{x} I_{\infty}^{1-\mu} \Psi(x)\right|_{x=0}=0, \\
& \lim _{x \rightarrow \infty}{ }_{x} D_{\infty}^{\mu} \Psi(x)=0 .
\end{aligned}
$$

Theorem 4.4 The eigenvalues of SFSLP2 (43)-(44) are given by

$$
\lambda_{n}^{(\alpha, \mu)}=-\frac{\Gamma(n+\alpha+\mu)}{\Gamma(n+\alpha)}, \quad n=0,1,2, \ldots
$$

The corresponding eigenfunctions to different eigenvalues are orthogonal with respect to the weighted functions, $\omega_{2}^{(\alpha)}(x)=x^{\alpha-1} e^{x}$, and are given by

$$
\Psi_{n}^{(\alpha)}(x)=e^{-x} \mathcal{L}_{n}^{(\alpha-1)}(x), \quad n=0,1,2, \ldots
$$

Proof First we prove that the generalized Laguerre functions $\Psi_{n}^{(\alpha)}$ in (45) obey the boundary conditions (44). Using (36) and (37), we have

$$
\begin{aligned}
\left.x^{\alpha+\mu-1} e^{x}{ }_{x} I_{\infty}^{1-\mu}\left[e^{-x} \mathcal{L}_{n}^{(\alpha-1)}(x)\right]\right|_{x=0} & =\left.x^{\alpha+\mu-1} e^{x}\left[e^{-x} \mathcal{L}_{n}^{(\alpha+\mu)}(x)\right]\right|_{x=0} \\
& =\left.x^{\alpha+\mu-1} \mathcal{L}_{n}^{(\alpha+\mu)}(x)\right|_{x=0}=0
\end{aligned}
$$

and

$$
\lim _{x \rightarrow \infty}{ }_{x} D_{\infty}^{\mu}\left[e^{-x} \mathcal{L}_{n}^{(\alpha-1)}(x)\right]=\lim _{x \rightarrow \infty}-e^{-x} \mathcal{L}_{n}^{(\alpha+\mu-1)}(x)=0 .
$$

Next, substituting the function $\Psi_{n}^{(\alpha)}$ in (45) in equation (43) yields

$$
\begin{aligned}
{ }_{0}^{C} D_{x}^{\mu}\left[x^{\alpha+\mu-1} e^{x}{ }_{x}^{R} D_{\infty}^{\mu}\left(e^{-x} \mathcal{L}_{n}^{(\alpha-1)}(x)\right)\right] & ={ }_{0}^{C} D_{x}^{\mu}\left[x^{\alpha+\mu-1} e^{x} e^{-x} \mathcal{L}_{n}^{(\alpha+\mu-1)}(x)\right] \\
& ={ }_{0}^{C} D_{x}^{\mu}\left[x^{\alpha+\mu-1} \mathcal{L}_{n}^{(\alpha+\mu-1)}(x)\right] \\
& =\frac{\Gamma(n+\alpha+\mu)}{\Gamma(n+\alpha)} x^{\alpha-1} \mathcal{L}_{n}^{(\alpha-1)}(x) \\
& =-\lambda_{n}^{(\alpha, \mu)} \omega_{2}^{(\alpha, \mu)}(x) \Psi_{n}^{(\alpha)}(x),
\end{aligned}
$$

where the corresponding eigenvalues are given by

$$
\lambda_{n}^{(\alpha, \mu)}=-\frac{\Gamma(n+\alpha+\mu)}{\Gamma(n+\alpha)} .
$$


Finally, we show that the eigenfunctions (51) are orthogonal with respect to $\omega_{2}^{(\alpha)}(x)=x^{\alpha-1} e^{x}$ as follows:

$$
\begin{aligned}
\int_{0}^{\infty} \Psi_{m}^{(\alpha)}(x) \Psi_{n}^{(\alpha, \mu)}(x) \omega_{2}^{(\alpha)}(x) d x & =\int_{0}^{\infty} e^{-2 x} \mathcal{L}_{n}^{(\alpha-1)}(x) \mathcal{L}_{m}^{(\alpha-1)}(x) x^{\alpha-1} e^{x} d x \\
& =\int_{0}^{\infty} \mathcal{L}_{n}^{(\alpha-1)}(x) \mathcal{L}_{m}^{(\alpha-1)}(x) x^{\alpha-1} e^{-x} d x \\
& =\frac{\Gamma(n+\alpha)}{\Gamma(n+1)} \delta_{m n}
\end{aligned}
$$

which completes the proof.

Analogously, we derive in the following theorem the eigenvalues and eigenfunctions of SFSLP3 (21)-(22) with $p_{3}(x)=x^{1-\alpha} e^{-x}$ and $\omega_{3}^{(\alpha)}(x)=x^{\alpha-1} e^{x}$.

$$
{ }_{x}^{R} D_{\infty}^{\mu}\left(x^{1-\alpha} e^{-x}{ }_{0} D_{x}^{\mu} f(x)\right)+\lambda x^{\alpha-1} e^{-x} f(x)=0, \quad 0 \leq x<\infty,
$$

subject to

$$
\begin{aligned}
& f(0)=0, \\
& \lim _{x \rightarrow \infty} I_{\infty}^{1-\mu}\left[x^{1-\alpha} e^{-x C} D_{x}^{\mu} f(x)\right]=0 .
\end{aligned}
$$

For SFSLP4 (23)-(24) with $p_{4}(x)=x^{\alpha+\mu-1} e^{x}$ and $\omega_{4}^{(\alpha)}(x)=x^{1-\alpha-\mu} e^{x}$, the proofs are straightforward and similar to Theorem 4.3 and Theorem 4.4 and we omit them. Now, we consider

$$
{ }_{0}^{R} D_{x}^{\mu}\left(x^{\alpha+\mu-1} e^{x}{ }_{x} D_{\infty}^{\mu} f(x)\right)+\lambda x^{1-\alpha-\mu} e^{x} f(x)=0, \quad 0 \leq x<\infty,
$$

subject to

$$
\begin{aligned}
& \left.{ }_{0} I_{x}^{1-\mu}\left[x^{\alpha+\mu-1} e_{x}^{x C} D_{\infty}^{\mu} f(x)\right]\right|_{x=0}=0, \\
& \lim _{x \rightarrow \infty} f(x)=0 .
\end{aligned}
$$

Theorem 4.5 1. The eigenvalues of SFSLP3 (46)-(47) are given by

$$
\lambda_{n}^{(\alpha, \mu)}=-\frac{\Gamma(n+\alpha+\mu)}{\Gamma(n+\alpha)}, \quad n=0,1,2, \ldots
$$

The corresponding eigenfunctions to different eigenvalues are orthogonal with respect to the weighted functions, $\omega_{3}^{(\alpha)}(x)=x^{\alpha-1} e^{-x}$, and are given by

$$
f(x)=\Phi_{n}^{(\alpha, \mu)}(x)=x^{\alpha+\mu-1} \mathcal{L}_{n}^{(\alpha+\mu-1)}(x), \quad n=0,1,2, \ldots
$$

The eigenvalues of SFSLP4 (48)-(49) are given by

$$
\lambda_{n}^{(\alpha, \mu)}=-\frac{\Gamma(n+\alpha+\mu)}{\Gamma(n+\alpha)}, \quad n=0,1,2, \ldots
$$


The corresponding eigenfunctions to different eigenvalues are orthogonal with respect to the weighted functions, $\omega_{4}^{(\alpha, \mu)}(x)=x^{1-\alpha-\mu} e^{x}$, and are given by

$$
f(x)=\Psi_{n}^{(\alpha)}(x)=e^{-x} \mathcal{L}_{n}^{(\alpha-1)}(x), \quad n=0,1,2, \ldots
$$

Remark 1 We obtain two types of eigenfunctions for four classes of SFSLPs. The first one is given by $\Phi_{n}^{(\alpha, \mu)}(x)=x^{\alpha+\mu-1} \mathcal{L}_{n}^{(\alpha+\mu-1)}(x)$, which we call the generalized Laguerre polynomials (GLPS), and the second one is given by $\Psi_{n}^{(\alpha)}(x)=e^{-x} \mathcal{L}_{n}^{(\alpha-1)}(x)$, which we call the generalized Laguerre functions (GLFs). In contrast to the GLPs, the GLFs are well behaved at infinity, due to the exponential decay term. Moreover, thanks to the self-adjointness of SFSLOs, we have real and discrete eigenvalues.

We turn to the orthogonal basis for the weighted Hilbert space $L_{\omega^{(\alpha, \mu)}}^{2}(0, \infty)$, where $\omega^{(\alpha, \mu)}=x^{1-\alpha-\mu} e^{-x}$ belongs to the most important properties of our eigenfunctions. In order to prove that the obtained eigenfunctions $\Phi_{n}^{(\alpha, \mu)}(x)$ in (41) form an orthogonal basis for $L_{\omega_{i}^{(\alpha, \mu)}}^{2}(0, \infty)$, the orthogonality of eigenfunctions is proved in Theorem 4.3. Hence, we only need to prove the completeness of the eigenfunctions $\Phi_{n}^{(\alpha, \mu)}(x)$.

The completeness of a system of functions on a compact interval can be straightforwardly proved, while the difficult cases are dealing with the system of functions on unbounded intervals. However, there is a nice trick by von Neumann that uses the generating function (32) of the Laguerre polynomial to permit the reduction to a bounded interval (for relevant results, see [15], p.108).

We present below the completeness of the GLFs $\Phi_{n}^{(\alpha, \mu)}(x)$ in (41) and also the completeness of the GLF $\Psi_{n}^{(\alpha)}(x)$ in (45) on the half-line.

Theorem 4.6 For $\alpha, \mu \in(0,1)$, we have the following:

1. The GLPS

$$
\Phi_{n}^{(\alpha, \mu)}(x)=x^{\alpha+\mu-1} \mathcal{L}_{n}^{(\alpha+\mu-1)}(x), \quad n=0,1,2, \ldots
$$

form a complete subspace of $L_{\omega^{(\alpha, \mu)}}^{2}(0, \infty), \omega^{(\alpha, \mu)}(x)=x^{1-\alpha-\mu} e^{-x}$.

2. The GLFs

$$
\Psi_{n}^{(\alpha)}(x)=e^{-x} \mathcal{L}_{n}^{(\alpha-1)}(x), \quad n=0,1,2, \ldots
$$

form a complete subspace of $L_{\omega^{(\alpha)}}^{2}(0, \infty), \omega^{(\alpha)}(x)=x^{\alpha-1} e^{x}$.

Proof Proving that $\Phi_{n}^{(\alpha, \mu)}(x)$ is complete in $L_{\omega^{\alpha, \mu}}^{2}(0, \infty)$ is equivalent to proving that $\widetilde{\Phi}_{n}^{(\alpha, \mu)}(x):=x^{\frac{1-\alpha-\mu}{2}} e^{-\frac{x}{2}} \Phi_{n}^{(\alpha, \mu)}(x)$ is complete in $L^{2}(0, \infty)$. Now, by setting $y=e^{-x}$, we will prove instead that

$$
\widetilde{\Phi}_{n}^{(\alpha, \mu)}(x)=\left(\ln \frac{1}{y}\right)^{\frac{\alpha+\mu-1}{2}} \sqrt{y}, \mathcal{L}_{n}^{(\alpha+\mu-1)}\left(\ln \frac{1}{y}\right)=\widetilde{\Phi}_{n}^{(\alpha, \mu)}(y)
$$

are complete in $L^{2}(0,1)$.

Since the polynomials are dense in $L^{2}(0,1)$ and $\left(\ln \frac{1}{y}\right)^{\frac{\alpha+\mu-1}{2}} \sqrt{y} \in L^{2}(0,1)$ for $\alpha+\mu>0$, we deduce that the functions $Q_{m}^{(\alpha, \mu)}(y)=\left(\ln \frac{1}{y}\right)^{\frac{\alpha+\mu-1}{2}} \sqrt{y} y^{m}$ span a dense subspace of $L^{2}(0,1)$. 
Hence, it is sufficient to prove that the closed linear space, determined by $\widetilde{\Phi}_{n}^{(\alpha, \mu)}(y)$, includes the functions $Q_{m}^{(\alpha, \mu)}(y)$. If $c_{n}$ is the $n$th generalized Laguerre coefficient of $Q_{m}^{(\alpha, \mu)}(y)$, we have to prove that

$$
\begin{aligned}
& \int_{0}^{1}\left[Q_{m}^{(\alpha, \mu)}(y)-\sum_{n=0}^{N} \widetilde{\Phi}_{n}^{(\alpha, \mu)}(y)\right]^{2} d y \\
& \quad=\int_{0}^{1}\left[\left(\ln \frac{1}{y}\right)^{\frac{\alpha+\mu-1}{2}} \sqrt{y}\left(y^{m}-\sum_{n=0}^{N} c_{n} \mathcal{L}_{n}^{(\alpha+\mu-1)}\left(\ln \frac{1}{y}\right)\right)\right]^{2} d y \rightarrow 0,
\end{aligned}
$$

as $N \rightarrow \infty$. By transforming back to $(0, \infty)$, this reduces the task to showing that

$$
\int_{0}^{\infty} x^{\alpha+\mu-1} e^{-x}\left[e^{-m x}-\sum_{n=0}^{N} c_{n} \mathcal{L}_{n}^{(\alpha+\mu-1)}(x)\right]^{2} d x \longrightarrow 0
$$

as $N \rightarrow \infty$. Thanks to the generating function in (32),

$$
\frac{e^{-\frac{t}{1-t} x}}{(1-t)^{\alpha+\mu}}=\sum_{n=0}^{\infty} t^{n} \mathcal{L}_{n}^{(\alpha+\mu-1)}(x), \quad|t|<1 .
$$

By taking $t=\frac{m}{m+1}$, we obtain $e^{-\frac{t}{1-t} x}=e^{-m x}$. Now choosing $c_{n}=(1-t)^{\alpha+\mu} t^{n}$ and substituting it into (54) yields

$$
\begin{aligned}
& \int_{0}^{\infty} x^{\alpha+\mu-1} e^{-x}\left[e^{-m x}-\sum_{n=0}^{N} c_{n} \mathcal{L}_{n}^{(\alpha+\mu-1)}(x)\right]^{2} d x \\
& =\int_{0}^{\infty} x^{\alpha+\mu-1} e^{-x}\left[(1-t)^{\alpha+\mu} \sum_{n=N+1}^{\infty} t^{n} \mathcal{L}_{n}^{(\alpha+\mu-1)}(x)\right]^{2} d x .
\end{aligned}
$$

Since the summation is convergent, the last expression becomes arbitrarily small when $N$ is sufficiently large. Therefore, the system $\widetilde{\Phi}_{n}^{(\alpha, \mu)}(x)$ is complete in $L^{2}(0, \infty)$, and we complete the proof of (52).

The second part (53) can be obtained in a similar manner.

\section{Approximation results by the generalized Laguerre functions}

In this section, we present some approximation properties of the GLFs. Let us first define the finite dimensional approximation space

$$
F_{N}^{\mu}\left(\mathbb{R}^{+}\right)=\left\{x^{\mu} \psi, \psi \in \mathbb{P}_{N}, \mu \in(0,1)\right\},
$$

where $\mathbb{P}_{N}$ is the set of all polynomials of degree at most $N$.

Consider the orthogonal projection $\pi_{N}^{\mu}: L_{\omega^{(\alpha, \mu)}}^{2}\left(\mathbb{R}^{+}\right) \rightarrow F_{N}^{\mu}\left(\mathbb{R}^{+}\right)$defined by

$$
\left\langle\pi_{N}^{\mu} u-u, v\right\rangle_{\omega^{(\alpha, \mu)}}=0, \quad \forall v \in F_{N}^{\mu}\left(\mathbb{R}^{+}\right),
$$

where $\langle f, g\rangle_{\omega^{(\alpha, \mu)}}=\int_{0}^{\infty} f(x) g(x) \omega^{(\alpha, \mu)} d x$. 
Thanks to the orthogonality, we can write

$$
\left(\pi_{N}^{\mu} u\right)(x)=\sum_{n=0}^{N} \hat{u}_{n}^{(\alpha, \mu)} \Phi_{n}^{(\alpha, \mu)}(x)
$$

where $\hat{u}_{n}^{(\alpha, \mu)}=\frac{\left\langle u, \Phi_{n}^{(\alpha, \mu)}\right\rangle_{\omega}(\alpha, \mu)}{\gamma_{n}^{(\alpha, \mu)}}, \gamma_{n}^{(\alpha, \mu)}=\frac{\Gamma(n+\alpha+\mu)}{\Gamma(n+1)}$.

In order to describe the $L_{\omega^{(\alpha, \mu)}}^{2}$-projection error, we introduce a weighted Sobolev space involving fractional derivatives. We have

$$
\begin{aligned}
& B_{\alpha, \mu}^{m, 1}\left(\mathbb{R}^{+}\right)=\left\{u:{ }_{0}^{C} D_{x}^{\mu+k} u \in L_{\omega^{(\alpha,-k)}}^{2}\left(\mathbb{R}^{+}\right), 0 \leq \mu+k \leq m, 0 \leq \mu \leq 1\right\}, \quad m \in \mathbb{N}_{0}, \\
& B_{\alpha, \mu}^{m, 2}\left(\mathbb{R}^{+}\right)=\left\{u:{ }_{x}^{C} D_{\infty}^{\mu+k} u \in L_{\omega^{(\alpha, k)}}^{2}\left(\mathbb{R}^{+}\right), 0 \leq \mu+k \leq m, 0 \leq \mu \leq 1\right\}, \quad m \in \mathbb{N}_{0},
\end{aligned}
$$

equipped with the norm and semi-norm

$$
\|u\|_{B_{\alpha, \mu}^{m, 1}}=\left[\sum_{k=0}^{m}\left\|{ }_{0}^{C} D_{x}^{\mu+k} u\right\|_{\omega^{(\alpha,-k)}}\right]^{\frac{1}{2}}, \quad|u|_{B_{\alpha, \mu}^{m, 1}}=\left\|{ }_{0}^{C} D_{x}^{\mu+k} u\right\|_{\omega^{(\alpha,-k)}},
$$

and

$$
\|u\|_{B_{\alpha, \mu}^{m, 2}}=\left[\sum_{k=0}^{m}\left\|{ }_{x}^{C} D_{\infty}^{\mu+k} u\right\|_{\omega^{(\alpha, k)}}\right]^{\frac{1}{2}}, \quad|u|_{B_{\alpha, \mu}^{m, 2}}=\left\|{ }_{x}^{C} D_{\infty}^{\mu+k} u\right\|_{\omega^{(\alpha, k)}} .
$$

We deduce from (34) and (33) that

$$
\begin{aligned}
{ }_{0}^{C} D_{x}^{\mu+k} \Phi_{n}^{(\alpha, \mu)}(x) & ={ }_{0}^{C} D_{x}^{\mu+k} x^{\alpha+\mu-1} \mathcal{L}_{n}^{(\alpha+\mu-1)}(x), \quad \text { with } k \leq \mu+k \leq k+1 \\
& =\frac{\Gamma(n+\alpha+\mu)}{\Gamma(n+\alpha-\mu-k-1)} x^{\alpha-k-1} \mathcal{L}_{n}^{(\alpha-k-1)}(x) .
\end{aligned}
$$

By using the above result, ${ }_{0}^{C} D_{x}^{\mu+k}, \Phi_{n}^{(\alpha, \mu)}(x)$ are orthogonal with respect to the weight $\omega^{(\alpha,-k)}=x^{1-\alpha+k} e^{-x}$, i.e.

$$
\begin{gathered}
\int_{0}^{\infty}{ }_{0}^{C} D_{x}^{\mu+k} \Phi_{m}^{(\alpha, \mu)}(x){ }_{0}^{C} D_{x}^{\mu+k} \Phi_{n}^{(\alpha, \mu)}(x) \omega^{(\alpha,-k)} d x \\
\quad=\left[\frac{\Gamma(n+\alpha+\mu)}{\Gamma(n+\alpha-\mu-k-1)}\right]^{2} \frac{\Gamma(n+\alpha-k)}{\Gamma(n+1)} \delta_{m n} .
\end{gathered}
$$

By summing (56) over $0 \leq k \leq m$, we obtain

$$
\sum_{k=0}^{m}\left\langle{ }_{0}^{C} D_{x}^{\mu+k} \Phi_{m}^{(\alpha, \mu)},{ }_{0}^{C} D_{x}^{\mu+k} \Phi_{n}^{(\alpha, \mu)}\right\rangle_{\omega^{(\alpha,-k)}}=0, \quad \forall m \neq n,
$$

which implies that $\Phi_{n}^{(\alpha, \mu)}$ are also orthogonal in $B_{\alpha, \mu}^{m, 1}\left(\mathbb{R}^{+}\right)$. 
Theorem 5.1 Let $u \in B_{\alpha, \mu}^{m, 1}\left(\mathbb{R}^{+}\right)$and $m \in \mathbb{N}_{0}$. Then we have

1. for $0 \leq k \leq m \leq N$,

$$
\left\|{ }_{0}^{C} D_{x}^{\mu+k}\left(\pi_{N}^{\mu} u-u\right)\right\|_{\omega^{(\alpha,-k)}} \leq N^{\frac{k-m}{2}}\left\|{ }_{0}^{C} D_{x}^{\mu+m} u\right\|_{\omega^{(\alpha,-m)}},
$$

2. for $0 \leq m \leq N$,

$$
\left\|\pi_{N}^{\mu} u-u\right\|_{\omega^{(\alpha, \mu)}} \leq N^{-\frac{m+\mu+2}{2}}\left\|{ }_{0}^{C} D_{x}^{\mu+m} u\right\|_{\omega^{(\alpha,-m)}}
$$

Proof By (55), we have

$$
{ }_{0}^{C} D_{x}^{\mu+k}\left(\pi_{N}^{\mu} u-u\right)=-\sum_{n=N+1}^{\infty} \hat{u}_{n}^{(\alpha, \mu) C} D_{x}^{\mu+k} \Phi_{n}^{(\alpha, \mu)}(x) .
$$

Hence, by the orthogonality (56), we get

$$
\left\|{ }_{0}^{C} D_{x}^{\mu+k}\left(\pi_{N}^{\mu} u-u\right)\right\|_{\omega^{(\alpha,-k)}}^{2}=\sum_{n=N+1}^{\infty}\left[\frac{\Gamma(n+\alpha+\mu)}{\Gamma(n+\alpha-k-1)}\right]^{2} \frac{\Gamma(n+\alpha-k)}{\Gamma(n+1)}\left|\hat{u}_{n}^{(\alpha, \mu)}\right|^{2} .
$$

In particular, we have

$$
\left\|{ }_{0}^{C} D_{x}^{\mu+k} u\right\|_{\omega^{(\alpha,-k)}}^{2}=\sum_{n=0}^{\infty}\left[\frac{\Gamma(n+\alpha+\mu)}{\Gamma(n+\alpha-k-1)}\right]^{2} \frac{\Gamma(n+\alpha-k)}{\Gamma(n+1)}\left|\hat{u}_{n}^{(\alpha, \mu)}\right|^{2} .
$$

Now, we obtain

$$
\begin{aligned}
\left\|{ }_{0}^{C} D_{x}^{\mu+k}\left(\pi_{N}^{\mu} u-u\right)\right\|_{\omega^{(\alpha,-k)}}^{2} & \\
\leq & \max _{n>N}\left\{\left[\frac{\Gamma(n+\alpha+\mu)}{\Gamma(n+\alpha-k-1)}\right]^{2} \frac{\Gamma(n+\alpha-k)}{\Gamma(n+1)}\left[\frac{\Gamma(n+\alpha-m-1)}{\Gamma(n+\alpha+\mu)}\right]^{2} \frac{\Gamma(n+1)}{\Gamma(n+\alpha-m)}\right\} \\
& \cdot \sum_{n=N+1}^{\infty}\left[\frac{\Gamma(n+\alpha+\mu)}{\Gamma(n+\alpha-m-1)}\right]^{2} \frac{\Gamma(n+\alpha-m)}{\Gamma(n+1)}\left|\hat{u}_{n}^{(\alpha, \mu)}\right|^{2} \\
\leq & \max _{n>N}\left\{\left[\frac{\Gamma(n+\alpha-m-1)}{\Gamma(n+\alpha-k-1)}\right]^{2} \frac{\Gamma(n+\alpha-k)}{\Gamma(n+\alpha-m)}\right\}\left\|{ }_{0}^{C} D_{x}^{\mu+m} u\right\|_{\omega^{(\alpha,-m)}}^{2} .
\end{aligned}
$$

By the Stirling equation,

$$
\Gamma(x+1) \sim \sqrt{2 \pi} x^{x+\frac{1}{2}} e^{-x}, \quad x \gg 1,
$$

we have

$$
\frac{\Gamma(n+\alpha-m-1)}{\Gamma(n+\alpha-k-1)} \sim n^{k-m} \text { and } \quad \frac{\Gamma(n+\alpha-k)}{\Gamma(n+\alpha-m)} \sim n^{m-k} \quad \text { for } n \gg 1 .
$$

Hence,

$$
\left\|{ }_{0}^{C} D_{x}^{\mu+k}\left(\pi_{N}^{\mu} u-u\right)\right\|_{\omega^{(\alpha,-k)}}^{2} \lesssim N^{k-m}\left\|{ }_{0}^{C} D_{x}^{\mu+m} u\right\|_{\omega^{(\alpha,-m)}}^{2},
$$

which proves (58). 
The $L_{\omega^{(\alpha, \mu)}}^{2}$-projection error (59) can be obtained by using the same argument. We have

$$
\begin{aligned}
\left\|\pi_{N}^{\mu} u-u\right\|_{\omega^{(\alpha, \mu)}}^{2}= & \sum_{n=N+1}^{\infty}\left|\hat{u}_{n}^{(\alpha, \mu)}\right|^{2} \frac{\Gamma(n+\alpha+\mu)}{\Gamma(n+1)} \\
= & \sum_{n=N+1}^{\infty}\left|\hat{u}_{n}^{(\alpha, \mu)}\right|^{2}\left[\frac{\Gamma(n+\alpha+\mu)}{\Gamma(n+\alpha-m-1)}\right]^{2} \frac{\Gamma(n+\alpha-\mu)}{\Gamma(n+1)} \\
& \cdot\left[\frac{\Gamma(n+\alpha-m-1)}{\Gamma(n+\alpha+\mu)}\right]^{2} \frac{\Gamma(n+\alpha+\mu)}{\Gamma(n+\alpha-m)} \\
\leq & N^{-(m+\mu+2)}\left\|_{0}^{C} D_{x}^{\mu+m} u\right\|_{\omega^{(\alpha,-m)}}^{2},
\end{aligned}
$$

which implies (59).

Theorem 5.2 Let $u \in B_{\alpha, \mu}^{m, 2}\left(\mathbb{R}^{+}\right)$and $m \in \mathbb{N}_{0}$. Then we have

1. for $0 \leq k \leq m \leq N$,

$$
\left\|{ }_{x}^{C} D_{\infty}^{\mu+k}\left(\pi_{N}^{\mu} u-u\right)\right\|_{\omega^{(\alpha, k)}} \leq N^{\frac{k-m}{2}}\left\|{ }_{x}^{C} D_{\infty}^{\mu+m} u\right\|_{\omega^{(\alpha, m)}}
$$

2. for $0 \leq m \leq N$,

$$
\left\|\pi_{N}^{\mu} u-u\right\|_{\omega^{(\alpha)}} \leq N^{-\frac{m+\mu+2}{2}}\left\|{ }_{x}^{C} D_{\infty}^{\mu+m} u\right\|_{\omega^{(\alpha, m)}} .
$$

Proof The proof is similar to that of Theorem 5.1 and thus omitted.

\section{The generalized Laguerre transform}

This section is based on the classical Laguerre transform by McCully [16]. We introduce the generalized Laguerre transform and use this transform to solve the fractional heat (diffusion) equation.

Definition 6.1 The generalized Laguerre transform of a function $F$ is defined by

$$
T\{F(x)\}=f^{(\alpha, \mu)}(n)=\int_{0}^{\infty} e^{-x} \mathcal{L}_{n}^{(\alpha+\mu-1)}(x) F(x) d x .
$$

The inverse transform $T^{-1}\left\{f^{(\alpha, \mu)}(n)\right\}$ of $T\{F(x)\}$ is given by

$$
F(x)=T^{-1}\left\{f^{(\alpha, \mu)}(n)\right\}=x^{\alpha+\mu-1} \sum_{n=0}^{\infty} \frac{n !}{\Gamma(n+\alpha+\mu)} f^{(\alpha, \mu)}(n) \mathcal{L}_{n}^{(\alpha+\mu-1)}(x) .
$$

Example 1 For $0<\alpha+\mu<2$, we have the following:

1. $T\{1\}=\frac{\Gamma(n+\alpha+\mu-1)}{n ! \Gamma(\alpha+\mu-1)} \quad$ (Equation (16.2.9) in [17]),

2. $T\left\{x^{\alpha+\mu-1}\right\}=0 \quad$ (Equation (124) in [13]),

3. $T\left\{e^{-s x}\right\}=\frac{1}{s+1} \sum_{k=0}^{n}\left(\begin{array}{c}n+\alpha+\mu \\ n-k\end{array}\right) \frac{(-1)^{k}}{(s+1)^{k}}, \quad s>1 \quad$ (Equation (125) in [13]), 
4. $T\left\{x^{\alpha+\mu-1} e^{-s x}\right\}=\frac{\Gamma(n+\alpha+\mu) s^{n}}{n !(s+1)^{n+\alpha+\mu}}, \quad s>-1 \quad$ (Equation (16.2.10) in [17]),

5. $T\left\{x^{\alpha+\mu+\beta-1}\right\}=\Gamma(\alpha+\mu+\beta) \sum_{k=0}^{\infty} \frac{(-1)^{k}}{k !} \frac{\Gamma(k+\beta)}{\Gamma(\beta)}, \quad \beta>0$

(Equation (16.2.15) in [17]).

Theorem 6.2 If $T\{F(x)\}=f^{(\alpha, \mu)}(n)$ exists and

1. F satisfies the boundary conditions (40), then

$$
T\left\{x^{\alpha+\mu-1} e^{x}{ }_{x}^{C} D_{\infty}^{\mu}\left[x^{1-\alpha} e^{-x}{ }_{0}^{R} D_{x}^{\mu} F(x)\right]\right\}=-\lambda_{n}^{(\alpha, \mu)} f^{(\alpha, \mu)}(n),
$$

2. F satisfies the boundary conditions (44), then

$$
T\left\{x^{\alpha+\mu-1} e^{x}{ }_{x}^{R} D_{\infty}^{\mu}\left[x^{1-\alpha} e^{-x}{ }_{0}^{C} D_{x}^{\mu} F(x)\right]\right\}=-\lambda_{n}^{(\alpha, \mu)} f^{(\alpha, \mu)}(n),
$$

where $\lambda_{n}^{(\alpha, \mu)}=\frac{\Gamma(n+\alpha+\mu)}{\Gamma(n+\mu)}$.

Proof First, by using the integration by parts equation (12), we obtain

$$
\begin{aligned}
T & \left\{x^{\alpha+\mu-1} e^{x}{ }_{x} D_{\infty}^{\mu}\left[x^{1-\alpha} e^{-x}{ }_{0}^{R} D_{x}^{\mu} F(x)\right]\right\} \\
= & \int_{0}^{\infty} x^{\alpha+\mu-1} \mathcal{L}_{n}^{(\alpha+\mu-1)}(x){ }_{x}^{C} D_{\infty}^{\mu}\left(x^{1-\alpha} e^{-x}{ }_{0}^{R} D_{x}^{\mu} F(x)\right) d x \\
= & \int_{0}^{\infty} x^{1-\alpha} e^{-x}{ }_{0}^{R} D_{x}^{\mu} F(x){ }_{0}^{R} D_{x}^{\mu}\left(x^{\alpha+\mu-1} \mathcal{L}_{n}^{(\alpha+\mu-1)}(x)\right) d x \\
& +\left.x^{1-\alpha} e_{0}^{-x R} D_{x}^{\mu} F(x){ }_{x} I_{\infty}^{1-\mu}\left(x^{\alpha+\mu-1} \mathcal{L}_{n}^{(\alpha+\mu-1)}(x)\right)\right|_{x=0} ^{\infty} \\
= & \frac{\Gamma(n+\alpha+\mu)}{\Gamma(n+\alpha)} \int_{0}^{\infty} e_{0}^{-x R} D_{x}^{\mu} F(x) \mathcal{L}_{n}^{(\alpha-1)}(x) d x .
\end{aligned}
$$

Applying the integration by parts equation (12) and using (36) and (37), we get

$$
\begin{aligned}
T & \left\{x^{\alpha+\mu-1} e^{x}{ }_{x} D_{\infty}^{\mu}\left[x^{1-\alpha} e^{-x}{ }_{0}^{R} D_{x}^{\mu} F(x)\right]\right\} \\
= & \frac{\Gamma(n+\alpha+\mu)}{\Gamma(n+\alpha)} \int_{0}^{\infty} F(x){ }_{x}^{C} D_{\infty}^{\mu}\left(e^{-x} \mathcal{L}_{n}^{(\alpha-1)}(x)\right) d x \\
& -\left.e^{-x} \mathcal{L}_{n}^{(\alpha-1)}(x)_{x} I_{\infty}^{1-\mu} F(x)\right|_{x=0} ^{\infty} \\
= & \frac{\Gamma(n+\alpha+\mu)}{\Gamma(n+\alpha)} \int_{0}^{\infty} F(x)_{x} I_{\infty}^{1-\mu}\left(-e^{-x} \mathcal{L}_{n}^{(\alpha-1)}(x)\right) d x \\
= & -\frac{\Gamma(n+\alpha+\mu)}{\Gamma(n+\alpha)} \int_{0}^{\infty} F(x) e^{-x} \mathcal{L}_{n}^{(\alpha+\mu-1)}(x) d x \\
= & -\lambda_{n}^{(\alpha, \mu)} f^{(\alpha, \mu)}(n) .
\end{aligned}
$$


The second part can be proved similarly, by using the integration by parts equation (11). We have

$$
\begin{aligned}
T & \left\{x^{\alpha+\mu-1} e^{x}{ }_{x}^{R} D_{\infty}^{\mu}\left[x^{1-\alpha} e^{-x}{ }_{0}{ }_{0} D_{x}^{\mu} F(x)\right]\right\} \\
= & \int_{0}^{\infty} x^{\alpha+\mu-1} \mathcal{L}_{n}^{(\alpha+\mu-1)}(x){ }_{x}^{R} D_{\infty}^{\mu}\left[x^{1-\alpha} e^{-x}{ }_{0} D_{x}^{\mu} F(x)\right] d x \\
= & \int_{0}^{\infty} x^{1-\alpha} e^{-x}{ }_{0}^{C} D_{x}^{\mu} F(x){ }_{0}^{C} D_{x}^{\mu}\left[x^{\alpha+\mu-1} \mathcal{L}_{n}^{(\alpha+\mu-1)}(x)\right] d x \\
& -\left.x^{\alpha+\mu-1} \mathcal{L}_{n}^{(\alpha+\mu-1)}(x)_{x} I_{\infty}^{1-\mu}\left[x^{1-\alpha} e^{-x}{ }_{0}^{C} D_{x}^{\mu} F(x)\right]\right|_{x=0} ^{\infty} \\
= & -\frac{\Gamma(n+\alpha+\mu)}{\Gamma(n+\alpha)} \int_{0}^{\infty} e^{-x}{ }_{0} D_{x}^{\mu} F(x) \mathcal{L}_{n}^{(\alpha-1)}(x) d x .
\end{aligned}
$$

The last expression is obtained by replacing ${ }_{0}^{C} D_{x}^{\mu}$ by ${ }_{0}^{R} D_{x}^{\mu}$ and using (34). Applying the integration by parts equation (11) again and using (37), we get

$$
\begin{aligned}
T\left\{x^{\alpha+\mu-1} e^{x}{ }_{x}^{R} D_{\infty}^{\mu}\left[x^{1-\alpha} e^{-x}{ }_{0}^{C} D_{x}^{\mu} F(x)\right]\right\} \\
=-\lambda_{n}^{(\alpha, \mu)}\left[\int_{0}^{\infty} F(x){ }_{x}^{R} D_{\infty}^{\mu}\left(e^{-x} \mathcal{L}_{n}^{(\alpha-1)}(x)\right) d x\right. \\
\left.\quad+\left.F(x)_{x} I_{\infty}^{1-\mu}\left(e^{-x} \mathcal{L}_{n}^{(\alpha-1)}(x)\right)\right|_{x=0} ^{\infty}\right] \\
=-\lambda_{n}^{(\alpha, \mu)} f^{(\alpha, \mu)}(n) .
\end{aligned}
$$

Example 2 (The space-fractional heat transfer problem of type 1) We consider the one dimension heat transfer in a semi-infinite medium, which is described by the following fractional partial differential equation with order $\mu \in(0,1)$ :

$$
c \rho U_{t}(x, t)={ }_{x}^{C} D_{\infty}^{\mu}\left[\kappa{ }_{0}^{R} D_{x}^{\mu} U(x, t)\right]+Q(x, t), \quad 0 \leq x<\infty, t>0,
$$

where $c=x^{1-\alpha-\mu}, \rho=e^{-x}$, and $\kappa=x^{1-\alpha} e^{-x}$ are the thermal coefficients. The source $Q(x, t)=$ $e^{-x} F(t)$. Therefore, SFSLP1 describing this semi-infinite fractional heat transfer is

$$
U_{t}(x, t)=x^{\alpha+\mu-1} e^{x}{ }_{x} D_{\infty}^{\mu}\left[x^{1-\alpha} e^{-x}{ }_{0}^{R} D_{x}^{\mu}(U(x, t))\right]+x^{\alpha+\mu-1} F(t) .
$$

If the medium is insulated laterally, the corresponding boundary conditions and initial condition are

$$
\begin{aligned}
& \left.x^{1-\alpha} e_{0}^{-x} I_{x}^{1-\mu} U(x, t)\right|_{x=0}=0, \quad \lim _{x \rightarrow \infty} x^{1-\alpha} e^{-x} I_{x} I_{x}^{-\mu} U(x, t)=0, \quad t>0, \\
& U(x, 0)=G(x), \quad 0 \leq x<\infty .
\end{aligned}
$$

Applying the generalized Laguerre transform with respect to $x$ to (64) and using (62), we obtain

$$
\frac{d}{d t} u^{(\alpha, \mu)}(n, t)=-\lambda_{n}^{(\alpha, \mu)} u^{(\alpha, \mu)}(n, t), \quad n=1,2, \ldots
$$


When $n=0$, we get

$$
\frac{d}{d t} u^{(\alpha, \mu)}(0, t)=-\lambda_{0}^{(\alpha, \mu)} u^{(\alpha, \mu)}(0, t)+\Gamma(\alpha+\mu) F(t)
$$

with the initial condition $u^{(\alpha, \mu)}(n, 0)=T\{G(x)\}:=g^{(\alpha, \mu)}(n)$. We have

$$
\begin{aligned}
& u^{(\alpha, \mu)}(n, t)=g^{(\alpha, \mu)}(n) e^{-\lambda_{n}^{(\alpha, \mu)} t}, \quad n=1,2, \ldots \\
& u^{(\alpha, \mu)}(0, t)=e^{-\lambda_{0}^{(\alpha, \mu)} t}\left[g^{(\alpha, \mu)}(0)+\Gamma(\alpha+\mu) \int_{0}^{t} F(\tau) e^{\lambda_{0}^{(\alpha, \mu)} \tau} d \tau\right] .
\end{aligned}
$$

The inverse generalized Laguerre transform leads to the solution

$$
\begin{aligned}
U(x, t)= & e^{-\lambda_{0}^{(\alpha, \mu)} t}\left[g^{(\alpha, \mu)}(0)+\Gamma(\alpha+\mu) \int_{0}^{t} F(\tau) e^{\lambda_{0}^{(\alpha, \mu)} \tau} d \tau\right] \frac{x^{\alpha+\mu-1}}{\Gamma(\alpha+\mu)} \\
& +x^{\alpha+\mu-1} \sum_{n=1}^{\infty} \frac{n !}{\Gamma(n+\alpha+\mu)} g^{(\alpha, \mu)}(n) e^{-\lambda_{n}^{(\alpha, \mu)} t} \mathcal{L}_{n}^{\alpha+\mu-1}(x) .
\end{aligned}
$$

We note that the solution of the fractional heat transfer problem coincides with the solution obtained from the integer-order heat transfer problem (see [16]) with $\alpha=0$ and $\mu=1$.

Example 3 (The space fractional heat conduction problem of type 2) Analogous to the previous example, we consider the one dimension heat transfer in a semi-infinite medium, which is described by SFSLP2. We have

$$
U_{t}(x, t)=x^{\alpha+\mu-1} e^{x}{ }_{x}^{R} D_{\infty}^{\mu}\left[x^{1-\alpha} e_{0}^{-x} D_{x}^{\mu}(U(x, t))\right]+x^{\alpha+\mu-1} F(t)
$$

with the corresponding boundary conditions and initial condition

$$
\begin{aligned}
& U(0, t)=0, \quad \lim _{x \rightarrow \infty}{ }_{x} I_{\infty}^{1-\mu}\left[x^{1-\alpha} e^{-x}{ }_{0}^{C} D_{x}^{\mu} U(x, t)\right]=0, \quad t>0, \\
& U(x, 0)=G(x), \quad 0 \leq x<\infty .
\end{aligned}
$$

Applying the generalized Laguerre transform with respect to $x$ to (65), we obtain the solution, which coincides with the solution of the space fractional heat conduction problem of type 1.

\section{Acknowledgements}

The author would to thank the reviewers as well as the editor for their helpful comments and suggestions, which significantly improve the quality of this article.

Competing interests

The author declares that he has no competing interests.

Authors' contributions

The author wrote this paper. The author read and approved the final manuscript.

\section{Publisher's Note}

Springer Nature remains neutral with regard to jurisdictional claims in published maps and institutional affiliations. 


\section{References}

1. Podlubny, I: Fractional Differential Equations. Academic Press, San Diego (1999)

2. Kilbas, AA, Srivastava, HM, Trujillo, JJ: Theory and Applications of Fractional Differential Equations. Elsevier, Amsterdam (2006)

3. Carpinteri, A, Mainardi, F: Fractals and Fractional Calculus in Continuum Mechanics. Springer, Berlin (1998)

4. Klimek, M, Agrawal, OP: On a regular fractional Sturm-Liouville problem with derivatives of order in $(0,1)$. In: Proceedings of 13th International Carpathian Control Conference, ICCC (2012)

5. Klimek, M, Agrawal, OP: Fractional Sturm-Liouville problem. Comput. Math. Appl. 66, 795-812 (2013)

6. Zayernouri, M, Karniadakis, GE: Fractional Sturm-Liouville eigen-problems: theory and numerical approximation. J. Comput. Phys. 252, 495-517 (2013)

7. Rivero, M, Trujillo, JJ, Velasco, MP: A fractional approach to the Sturm-Liouville problem. Cent. Eur. J. Phys. 11, 1246-1254 (2013)

8. Ansari, A: On finite fractional Sturm-Liouville transforms. Integral Transforms Spec. Funct. 26, 51-64 (2015)

9. Bas, E, Metin, F: Fractional singular Sturm-Liouville operator for Coulomb potential. Adv. Differ. Equ., 2013,300 (2013)

10. Bas, E, Yilmazer, R, Panakhov, E: Fractional solution of Bessel equation with N-method. Sci. World J. 2013, 685695 (2013)

11. Bas, E: Fundamental spectral theory of fractional singular Sturm-Liouville operator. J. Funct. Spaces Appl. 2013 915830 (2013)

12. Zhang, W, Yi, M: Sturm-Liouville problem and numerical method of fractional diffusion equation on fractals. Adv. Differ. Equ. 2015, 217 (2015)

13. Khosravian-Arab, H, Dehghan, M, Eslahchi, MR: Fractional Sturm-Liouville boundary value problems in unbounded domains: theory and applications. J. Comput. Phys. 299, 526-560 (2015)

14. Andrews, GE, Askey, R, Roy, R: Special Functions. Encyclopedia of Mathematics and Its Applications. Cambridge University Press, Cambridge (1999)

15. Szegö, G: Orthogonal Polynomials. Am. Math. Soc., Providence (1975)

16. McCully, J: The Laguerre transform. SIAM Rev. 2(3), 185-191 (1960)

17. Debnath, L, Bhatta, D: Integral Transforms and Their Applications. CRC Press, Boca Raton (2015)

\section{Submit your manuscript to a SpringerOpen ${ }^{\circ}$ journal and benefit from:}

- Convenient online submission

Rigorous peer review

- Open access: articles freely available online

- High visibility within the field

- Retaining the copyright to your article

Submit your next manuscript at $\boldsymbol{\nabla}$ springeropen.com 